ANNALES

POLONICI MATHEMATICI

$95.2(2009)$

\title{
A DSM proof of surjectivity of monotone nonlinear mappings
}

\author{
by A. G. RAmm (Manhattan, KS)
}

Abstract. A simple proof is given of a basic surjectivity result for monotone operators. The proof is based on the dynamical systems method (DSM).

1. Introduction. It is well-known that a continuous monotone function $f: \mathbb{R} \rightarrow \mathbb{R}$ such that

$$
\lim _{|x| \rightarrow \infty} \frac{x f(x)}{|x|}=\infty
$$

is surjective, i.e., the equation $f(x)=y$ is solvable for any $y \in \mathbb{R}$. Indeed, the monotonicity of $f$ implies

$$
[f(x)-f(s)](x-s) \geq 0, \quad \forall x, s \in \mathbb{R} .
$$

Therefore, taking $y=0$ without loss of generality, one concludes from (1) that $f(x) \leq 0$ for $x \leq 0$ and $f(x) \geq 0$ for $x \geq 0$. Since $f$ is continuous, it follows that there is a point $x_{0}$ such that $f\left(x_{0}\right)=0$.

If $y \neq 0$ is an arbitrary real number, then the function $F(x)=f(x)-y$ satisfies inequality (2) with $F$ in place of $f$, provided that (2) holds for $f$. Condition (1) is also satisfied for $F$ if it holds for $f$ :

$$
\lim _{|x| \rightarrow \infty} \frac{x F(x)}{|x|}=\lim _{|x| \rightarrow \infty}\left(\frac{x f(x)}{|x|}-\frac{x y}{|x|}\right)=\infty .
$$

Conditions (1) and (2) are generalized for nonlinear mappings $F$ in a real Hilbert space $H$ as follows:

$$
\lim _{\|u\| \rightarrow \infty} \frac{(u, F(u))}{\|u\|}=\infty
$$

2000 Mathematics Subject Classification: 47A52, 47H07, 47J05.

Key words and phrases: dynamical systems method, monotone operators, surjectivity, nonlinear equations. 
and

$$
(F(u)-F(v), u-v) \geq 0 \quad \forall u, v \in H .
$$

Here $(u, v)$ stands for the inner product in $H$. Equations with monotone operators arise in many applications.

We want to prove that if $F$ is twice Fréchet differentiable and conditions (3)-(4) hold, then $F$ is surjective, i.e., the equation

$$
F(u)=h
$$

is solvable for every $h \in H$. This is a basic result in the theory of monotone operators (see, e.g., [1]), which can be proved without the assumption about twice Fréchet differentiability, but then its proof becomes considerably less simple. Our aim is to give a simple and short proof of this result. It is based on the dynamical systems method (DSM) developed in [2].

ThEOREM 1. Assume that $F: H \rightarrow H$ is a Fréchet differentiable mapping satisfying conditions (3), (4). Then equation (5) is solvable for any $h$.

REMARK 1. If in (4) one has a strict inequality for $u \neq v$, then the solution to (5) is unique.

Remark 2. Condition (4) and Fréchet differentiability imply that $A:=$ $F^{\prime}(u) \geq 0$ for all $u \in H$.

REMARK 3. The Fréchet differentiability assumption can be weakened to semicontinuity ([3], see also [1]), but then the proof loses its elementary character.

2. Proof. Let us formulate the steps of our proof.

STEP 1. For any $a=$ const $>0$ the equation

$$
F\left(u_{a}\right)+a u_{a}=h
$$

has a unique solution $u_{a}$.

SteP 2.

$$
\sup _{0<a<1}\left\|u_{a}\right\|<c, \quad c=\text { const }>0 .
$$

By $c$ we denote various constants independent of $a$.

STEP 3. Using (7), select a sequence $u_{n}=u_{a_{n}}, a_{n} \rightarrow 0$, weakly convergent in $H$ to an element $u$ :

$$
u_{n} \rightarrow u, \quad n \rightarrow \infty .
$$

From (6) and (8) it follows that

$$
F\left(u_{n}\right) \rightarrow h, \quad n \rightarrow \infty .
$$

From (8), (9) and (4) one concludes that $u$ solves (5). 
Let us give a detailed proof.

STEP 1. Consider the problem

$$
\dot{v}=-A_{a}^{-1}[F(v)+a v-h], \quad v(0)=0 .
$$

Here $\dot{v}:=\frac{d v}{d t}, A_{a}:=A+a I, A:=F^{\prime}(v)$. Problem (10) is a version of the DSM (see $[2$, p. 115]). We claim that:

(a) problem (10) has a unique global solution, that is, the solution defined for all $t \in[0, \infty)$,

(b) there exists $v(\infty):=\lim _{t \rightarrow \infty} v(t)$,

(c) $F(v(\infty))+a v(\infty)=h$.

Claim (a) follows from local solvability of problem (10) and a uniform (with respect to $t$ ) bound on the norm $\|v(t)\|$. This bound is obtained below (see (13)). The local solvability follows from the standard result on local solvability of a differential equation with Lipschitz right-hand side. Our asumption about twice differentiability of $F$ implies that the right-hand side of equation (10) is Lipschitz. For monotone $F$ the twice differentiability assumption can be considerably weakened (see $[2,3]$ ), but then the proof of the local solvability becomes more complicated.

Define

$$
\|F(v(t))+a v(t)-h\|=: g(t), \quad \dot{g}:=\frac{d g}{d t} .
$$

Using (10), one gets

$$
g \dot{g}=\left(\left(F^{\prime}(v)+a I\right) \dot{v}, F(v(t))+a v(t)-h\right)=-g^{2} .
$$

Thus

$$
g(t)=g(0) e^{-t}
$$

From (11) and (10) one deduces

$$
\|\dot{v}\| \leq \frac{g(0)}{a} e^{-t}
$$

where the estimate $\left\|A_{a}^{-1}\right\| \leq 1 / a$ was used. This estimate holds because $A=F^{\prime}(v(t)) \geq 0$ by the monotonicity of $F$. Integrating (12) from $t$ to infinity yields

$$
\|v(t)-v(\infty)\| \leq \frac{g(0)}{a} e^{-t}
$$

Note that if $\|\dot{v}\| \leq g(t)$ and $g(t) \in L^{1}(0, \infty)$, then $v(\infty)$ exists by the Cauchy criterion for the existence of a limit:

$$
\|v(t)-v(s)\| \leq \int_{s}^{t} g(\tau) d \tau \rightarrow 0, \quad t, s \rightarrow \infty, t>s .
$$


It follows from (12) that

$$
\lim _{t \rightarrow \infty}\|\dot{v}\|=0 \text {. }
$$

Therefore, passing to the limit $t \rightarrow \infty$ in (10), one gets

$$
0=-A_{a}^{-1}(v(\infty))[F(v(\infty))+a v(\infty)-h] .
$$

Applying the operator $A_{a}(v(\infty))$ to equation (15), one sees that $v(\infty)$ solves equation (6).

Uniqueness of the solution to (6) is easy to prove: if $v$ and $w$ solve (6), then

$$
F(v)-F(w)+a(v-w)=0, \quad a>0 .
$$

Multiply this equation by $v-w$, use the monotonicity of $F$ (see (4)), and conclude that $v=w$. Step 1 is completed.

SteP 2. Multiply (6) by $u_{a} /\left\|u_{a}\right\|$ to get

$$
\frac{\left(F\left(u_{a}\right), u_{a}\right)}{\left\|u_{a}\right\|}+a\left\|u_{a}\right\|=\frac{\left(h, u_{a}\right)}{\left\|u_{a}\right\|} .
$$

Since $a>0$ and $\left(h, u_{a}\right) /\left\|u_{a}\right\| \leq\|h\|$, one gets

$$
\frac{\left(F\left(u_{a}\right), u_{a}\right)}{\left\|u_{a}\right\|} \leq\|h\|
$$

From (17) and (3) the desired estimate (7) follows. Step 2 is completed.

SteP 3. Let us prove that (4), (8) and (9) imply (5). Let $\eta \in H$ be arbitrary, and $s>0$ be a small number. Note that $u_{n} \rightarrow u$ and $g_{n} \rightarrow g$ imply $\left(u_{n}, g_{n}\right) \rightarrow(u, g)$. Using (4), one gets

$$
\left(F\left(u_{n}\right)-F(u-s \eta), u_{n}-u+s \eta\right) \geq 0, \quad \forall \eta \in H, s>0 .
$$

Let $n \rightarrow \infty$ in (18). Then, using (8) and (9), one concludes that

$$
\begin{array}{rll}
(h-F(u-s \eta), s \eta) \geq 0 & \forall \eta \in H, s>0, & \text { or } \\
(h-F(u-s \eta), \eta) \geq 0 & \forall \eta \in H, s>0 . &
\end{array}
$$

Let $s \rightarrow 0$ and use the continuity of $F$. (Here hemicontinuity of $F$ would suffice.) Then (19) implies

$$
(h-F(u), \eta) \geq 0 \quad \forall \eta \in H .
$$

Taking $\eta=h-F(u)$ in $(20)$, one concludes that $F(u)=h$. Step 3 is completed. Theorem 1 is proved.

\section{References}

[1] K. Deimling, Nonlinear Functional Analysis, Springer, Berlin, 1985.

[2] A. G. Ramm, Dynamical Systems Method for Solving Operator Equations, Elsevier, Amsterdam, 2007. 
[3] A. G. Ramm, Dynamical systems method for solving nonlinear operator equations, Int. J. Appl. Math. Sci. 1 (2004), 97-110.

Mathematics Department

Kansas State University

Manhattan, KS 66506-2602, U.S.A.

E-mail: ramm@math.ksu.edu

Received 17.6.2008

and in final form 12.8.2008 